\title{
Empirical Article
}

\section{Cognitive and Academic Skills in Two Developmental Cohorts of Different Ability Level: A Mutualistic Network Perspective}

\author{
Silvana Mareva, ${ }^{*}$ The CALM team, and \\ Joni Holmes \\ Medical Research Council Cognition and Brain Sciences Unit, University of Cambridge, UK
}

\begin{abstract}
Mutualistic theories assume that the mastering of a skill, either cognitive or academic, supports and amplifies the development of other such abilities. The current study uses network science to model cross-sectional associations between cognitive and academic performance in two age-matched developmental cohorts. One cohort was a community sample drawn from the general school population, while the other included struggling learners. The community sample outperformed the struggling learners across all measures. Network models suggested that although the tasks were similarly interrelated across cohorts, there were some notable differences in association strength: Academic skills were more closely coupled in the community sample, while maths was more strongly related to cognitive skills in the struggling learners. We demonstrate the utility of network models as an analytic framework that is consistent with contemporary theories of learning difficulties and the nature of the relationship between cognitive and learning skills more broadly.
\end{abstract}

Keywords: Learning difficulties, Cognitive skills, Academic skills, Mutualism, Network models

\section{General Audience Summary}

Multiple theories attempt to explain why academic and cognitive performance are closely related. Most traditional theories assume a one-way perspective whereby specific cognitive skills constrain particular aspects of academic development. However, contemporary evidence suggests that links between cognitive skills and academic achievement are rarely specific and that the mastery of academic skills can also influence cognitive development. In this study, we use a novel statistical modelling approach consistent with the possibility that cognitive and academic skills might reciprocally influence one another, and we compare whether the same patterns of associations emerge for children drawn from the general school population and those who are struggling at school. Our modelling approach demonstrated that there are multiple direct links between cognitive and academic skills, which are broadly similar for both groups of children. Overall, we argue that researchers aiming to understand learning difficulties should consider that while cognitive skills can influence learning, the process of learning can also influence cognitive skills.

Poor academic achievement is associated with low employment rates (de Beer et al., 2014) and increased risks of mental health problems (Tempelaar et al., 2017). The need to minimise these risks and reduce associated societal and economic costs has driven decades of research that has attempted to understand and remediate learning problems. Multiple theories attempt to

* Correspondence concerning this article should be addressed to Silvana Mareva at MRC Cognition and Brain Sciences, Cambridge, UK. Contact: silvana.mareva@mrc-cbu.cam.ac.uk (S.M.). 
explain academic difficulties. The traditional, and arguably still dominant assumption, is that learning problems are caused by specific cognitive deficits (e.g., Bishop \& Snowling, 2004; Melby-Lervåg et al., 2012; Pennington \& Ozonoff, 1996; Szucs et al., 2013). While the unidirectional approach has generated insights that have helped guide practice and policy (e.g., Reid \& Fawcett, 2008), there are several challenges that it cannot easily accommodate. More contemporary theories acknowledge that there is a more complex developmental interplay between cognitive and academic skills (e.g., KarmiloffSmith, 2009; Peng \& Kievit, 2020).

\section{Challenges of the Unidirectional One-to-One Mapping Perspective}

Traditional theoretical models of learning difficulties have assumed that cognitive abilities provide the foundation for academic development. For example, phonological processing deficits observed among children with reading difficulties provide the basis for the hypothesis that reading difficulties are caused by poor phonological processing (Bishop \& Snowling, 2004; Clayton et al., 2020; Perfetti, 2007). Similarly, working memory deficits observed among children with specific maths problems provide support for the hypothesis that memory systems are critical for maths (Szucs et al., 2013). Such theories are appealing due to the simplicity of assuming a "core deficit" (Astle \& Fletcher-Watson, 2020) and their direct implications for intervention (e.g., phonological interventions for struggling readers, Bowyer-Crane et al., 2008).

Core deficit theories are challenged by evidence suggesting that similar learning difficulties may stem from different causes (i.e., equifinality). For example, reading difficulties are not wholly explained by phonological deficits. Some struggling readers have difficulties in applying letter-sound correspondences to decode words, the primary role ascribed to phonological processing in reading (Castles \& Friedmann, 2014). However, other poor readers have no difficulties with decoding, but instead, struggle with reading comprehension, an aspect of reading assumed to be supported by skills such as working memory (e.g., Cain et al., 2004). Similarly, data-driven explorations of the links between cognitive and academic skills suggest that children can arrive at similar profiles of learning impairment through multiple etiological routes: Children with comparable difficulties in both reading and maths can have different cognitive profiles, such as relatively more severe problems with phonological processing or working memory (Astle et al., 2019). Consistent with equifinality, multiple deficit theories suggest that risk factors spanning multiple levels (genes, brain, cognition, and behaviour) contribute probabilistically to neurodevelopmental difficulties (Pennington, 2006) and that shared risk factors contribute to comorbidity. This idea is supported by evidence that processing speed explains some of the comorbidity between dyslexia, dyscalculia, and Attention Deficit Hyperactivity Disorder (ADHD), while language difficulties contribute to comorbidity between dyscalculia and dyslexia (McGrath et al., 2011; Peterson et al., 2017).

\section{Bidirectional Dynamics}

A challenge to both single and multiple deficit models comes from studies showing that while cognitive skills influence academic skills, academic skills also shape cognitive development. Take our earlier example of the association between phonological processing and reading. Phonological awareness contributes to reading development, but it also benefits from reading instruction and exposure to text (Huettig et al., 2018; Nation \& Hulme, 2011). Similar reciprocal effects, where the development of a given academic skill predicts growth in cognitive performance and vice-versa, have been reported between working memory and reading and maths (Miller-Cotto \& Byrnes, 2020), IQ and reading (Ferrer et al., 2007, 2010), nonverbal reasoning and vocabulary (Kievit et al., 2017; Kievit, Hofman, \& Nation, 2019), and executive functions and maths (Schmitt et al., 2017; Van der Ven et al., 2012). These examples suggest that difficulties in one domain may have downstream effects on other abilities and challenge the use of analytic strategies in which cognitive deficits are uniquely modelled as predictors and academic skills as outcomes.

An alternative view of the relationship between cognitive and academic skills, consistent with evidence for reciprocal benefits, is mutualism. Mutualism proposes that different abilities interact bidirectionally to reinforce one another during development (Peng \& Kievit, 2020; Van Der Maas et al., 2006). In other words, the mastering of a skill supports and amplifies the development of other abilities. The mutualism model is neuro-constructivist in nature, acknowledging that specialised abilities likely emerge developmentally through a process of multidirectional interactions between genes, brain, cognition, and the environment (Karmiloff-Smith, 2009; Kievit, 2020).

In the context of cognitive-academic coupling, mutualistic transactions might be driven by experience, and particularly by educational experiences. Fundamental cognitive resources support the development of academic skills while performing academic tasks uses and trains cognitive abilities, and over time, these relationships become mutually beneficial (Peng \& Kievit, 2020). For example, fluid reasoning skills aid the use of analogies and abstract schema in academic tasks, while concrete knowledge (e.g., verbal skills) supports the decomposition of complex reasoning tasks (Kievit et al., 2017). The type and strength of these transactions might be moderated by experience. Weaker or absent bidirectional relations have been reported in children with learning difficulties (e.g., Ferrer et al., 2010; Quinn et al., 2019). This could be because difficulties with specific skills operate as the bottleneck to the development of the wider cognitive system and/or because struggling learners may choose to avoid exercises that tax their area(s) of weakness. In the latter case, the net result could be that poor learners may engage in fewer activities that develop positively reinforcing associations, which over time might constrain both cognitive and academic development. 


\section{Cognitive and Academic Skills: New Approaches}

To address the challenges outlined above, it is necessary to rethink the recruitment and analysis strategies typically used to study learning difficulties. The prevailing sampling approach involves recruiting highly selective samples of children based on the presence of a specific diagnosis or difficulty, with comorbidity often treated as a confound (e.g. Szucs et al., 2013). This runs counter to a wealth of evidence showing that disorders are highly comorbid, heterogeneous, and explained by multiple causes (Astle et al., 2019; Peters \& Ansari, 2019). Overstating the "purity" of learning problems at the point of recruitment biases outcomes towards core-deficit accounts. To avoid such biases, learning-related problems are increasingly studied using transdiagnostic approaches (Astle et al., 2021; Casey et al., 2014; Holmes et al., 2019; Mareva \& Holmes, 2019). These approaches aim to understand the processes and causes of difficulties that occur across individuals irrespective of diagnosis or group membership.

In terms of methods, unidirectional associations between cognition and academic performance are typically modelled using latent variable approaches whereby cognitive and academic factors are derived separately, and the relations between them are mapped as directional paths from cognitive to academic skills (Peterson et al., 2017). One alternative, which can better accommodate the possibility of equifinality without assuming causational directionality, comes from network science. Network models are relatively new to cognitive science but have already proved useful in tackling similar challenges in the field of psychopathology (Borsboom, 2016). In simple terms, network models use partial correlations to depict how each observed variable relates to all other observed variables. In this way, they offer a modelling framework that is more consistent with mutualism and the possibility that reciprocal mutual transactions are the generating process behind the relationships observed across cognitive and academic skills. Applied to cross-sectional data, these models offer a tool to explore or test specific hypotheses about whether and how the complex developmental interplay between academic and cognitive skills may differ across time points and/or groups of children.

\section{Objectives}

The aim of the current study was to apply networks models to explore and compare the interrelations between cognitive and academic abilities in a community sample and a sample of struggling learners. A heterogeneous cohort of children identified by practitioners as having school-related difficulties was included to represent struggling learners. The comparison cohort was an age-matched group of children selected as being nationally representative. For academic skills, the focus was on literacy and maths. For cognitive skills, we included assessments of processing speed, working memory, executive function, and nonverbal reasoning, all of which have been previously linked to academic performance in both typical and atypical learners (Altemeier et al., 2008; Booth et al., 2010; Gathercole et al., 2004; Geary, 2011; Green et al.,
2017; Holmes et al., 2020; Mayes \& Calhoun, 2007; Peng \& Fuchs, 2016; Taub et al., 2008; Yeniad et al., 2013). To our knowledge, this is the first application of network science to cognitive-academic interrelationships in learners of different abilities. Consistent with mutualism and equifinality, we anticipated there would be multiple direct links between cognitive and academic skills in both cohorts. We were agnostic as to whether and how task interrelationships would differ across cohorts.

\section{Method}

\section{Recruitment and Participants}

Children from two cohorts were included in this study: those from the Centre for Attention, Learning, and Memory (CALM) 800 and the Nathan-Klein Institute Rockland sample (NKI-RS). These two cohorts were chosen because they include identical tasks while having different ability levels. Recruitment details, inclusion criteria, ethical and testing procedures are described in the protocols of the respective cohorts (Holmes et al., 2019; Nooner et al., 2012). Briefly, CALM includes children referred by health and educational professionals for having difficulties with attention, learning, and/or memory. Some of the children had a diagnosed learning-related problem, others had multiple diagnoses, but the majority were undiagnosed despite coming to the attention of practitioners for struggling at school. The NKI-RS is a community cohort, demographically representative of the population of Rockland, New York. Further recruitment details for each cohort are provided in Supplement Section 1.

Due to testing sessions in the NKI-RS sample sometimes being more than a year apart, we subsampled children aged 8 to 18 years-old whose assessments were completed less than six months apart from each cohort $\left(N_{\mathrm{CALM}}=566, N_{\mathrm{NKI}}\right.$ $\mathrm{RS}=350$ ). This time window was chosen as a liberal estimate of the child being within the same developmental period for all assessments and is consistent with previous studies using the NKI-RS cohort (e.g., Simpson-Kent et al., 2020). To ensure the comparisons were not biased by differences in sample size, CALM participants were age-matched to NKI-RS children using propensity matching based on the nearest neighbour method (Ho et al., 2011). The final age-matched samples included 350 participants (CALM: $M_{\text {age }}=11.26, S D_{\text {age }}=2.21$, $69 \%$ male; NKI-RS: $M_{\text {age }}=11.99, S D_{\text {age }}=2.89,56 \%$ male). As expected, children with diagnosed neurodevelopmental problems were overrepresented in CALM (based on parent report, ADHD: 32\% CALM and 14\% NKI-RS; Learning problems (developmental language disorder, dyslexia, dysgraphia, or dyscalculia): 11\% CALM and 6\% NKI-RS; Autism Spectrum Disorders (ASD): $10 \%$ CALM and 0.3\% NKI-RS).

\section{Assessments}

Assessments that were available for both cohorts were included. All tasks were taken from standardised test batteries. The psychometric properties for each assessment, together with 
the standardised administration procedures, can be found in the associated testing kits. Brief descriptions of each assessment are provided below.

\section{Forward and Backward Digit Recall}

The forward and backward digit recall tasks from the Automated Working Memory Assessment (AWMA; Alloway, 2007) were administered to CALM participants, and the same tasks from the Wechsler Intelligence Scale for Children Revised (WISC-R; Wechsler, 1974) were administered to NKI-RS participants. In both cases, forward digit recall involved the serial recall of sequences of spoken digits, while backward digit recall required children to recall the digits in reverse serial order. The number of trials per sequence length was six for the AWMA and two for the WISC-R. The number of trials correct was scored and used in all analyses except for the permutation tests. These required identical measurement scales, so span was used to index performance for these analyses.

\section{Matrix Reasoning}

The Matrix Reasoning subtest of the Wechsler Abbreviated Scales of Intelligence II (WASI-II; Wechsler, 2011) was administered to children in both cohorts. Children are presented with increasingly complex nonverbal analogical reasoning problems in $2 \times 2$ matrices and are asked to select from a range of alternatives a shape that completes the pattern. The number of correctly solved matrices was scored.

\section{Motor Speed}

Children in both cohorts completed the Motor Speed test of the Delis Kaplan Executive Function System (D-KEFS; Delis et al., 2001). This measured time (in seconds) to trace a dotted line as quickly as possible. Completion time in seconds was scored.

\section{Tower}

The Tower subtest of the D-KEFS, completed by both cohorts, required children to move disks of different sizes around pegs from a start position to an end state shown on a picture following a set of rules. The number of correctly completed towers was scored.

\section{Trails Number-Letter Sequencing}

Both cohorts completed the Trails Number-Letter Sequencing task of the D-KEFS, which required children to connect letters and numbers in a progressive alternating sequence (e.g., 1A, 2-B, etc). Completion time in seconds was scored.

\section{Spelling, Reading, and Maths}

The Spelling, Word Reading, and Numerical Operations subtests of the Wechsler Individual Achievement Test II (WIAT-II; Wechsler, 2005) were administered to children in both cohorts to measure academic abilities. Numerical operations captured number identification, counting, and the ability to solve simple and complex maths problems. Spelling required children to write down individual letters and spell single words. Word reading involved identifying letters, matching sounds to letters, and reading single words of increasing complexity. To account for the large degree of overlap in the latter two scores (see Figure S1), they were combined into a single variable called literacy.

\section{Visual Scanning}

The D-KEFS Visual Scanning subtest was administered to children in both cohorts. Children were required to cross out all the number threes on a response page of numbers and letters as quickly as possible. Completion time in seconds was scored.

\section{Analysis Plan}

Network models were estimated to characterise the interrelations between cognitive and academic skills in each cohort. The accuracy of these results was scrutinised via a bootstrapping procedure. Permutation testing was then used to compare the two networks. Node strength and predictability were also estimated and compared. All statistical tests were performed in $\mathrm{R}(\mathrm{R}$ Core Team, 2020, see Supplement Section 2 for library details). In all analyses, raw scores were transformed such that higher values represented better performance. For most analyses apart from node predictability estimates, missing data were handled by estimating pairwise associations. To estimate node predictability and to check the robustness of the pairwise association method, all analyses were repeated following multiple

Table 1

Summary Statistics for CALM and NKI-RS Based on Raw Scores, Including Number of Complete Cases and Task Abbreviations for All Assessments

\begin{tabular}{|c|c|c|c|c|}
\hline \multirow[t]{2}{*}{ Task } & \multicolumn{2}{|c|}{ CALM } & \multicolumn{2}{|c|}{ NKI-RS } \\
\hline & $\mathbf{N}$ & M (SD) & $\mathbf{N}$ & M (SD) \\
\hline Backward Digit Recall (BDR)* & 348 & $11.44(4.25)$ & 301 & $5.28(2.04)$ \\
\hline Forward Digit Recall (FDR)* & 349 & $26.38(5.08)$ & 301 & $6.97(2.15)$ \\
\hline Numerical Operations (Maths). & 312 & $20.64(8.87)$ & 315 & $30.35(10.66)$ \\
\hline Matrix Reasoning (MxReas) & 350 & $13.46(5.52)$ & 315 & $18.30(4.64)$ \\
\hline Spelling and Reading (Lit) & 345 & $62.02(13.18)$ & 315 & $73.17(10.81)$ \\
\hline Visual Scanning (Scan) & 301 & $32.13(11.62)$ & 338 & $27.62(9.55)$ \\
\hline Motor Speed (Speed) & 299 & $40.14(17.13)$ & 338 & $32.44(16.20)$ \\
\hline Trails Number-Letter Sequencing (Switch) & 270 & $154.31(60.97)$ & 337 & $111.67(58.08)$ \\
\hline Tower & 279 & $14.44(3.80)$ & 339 & $15.46(3.76)$ \\
\hline
\end{tabular}

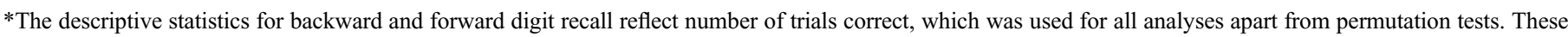
requires identical measurement scales, thus, for this analysis only, span was used to index performance. 


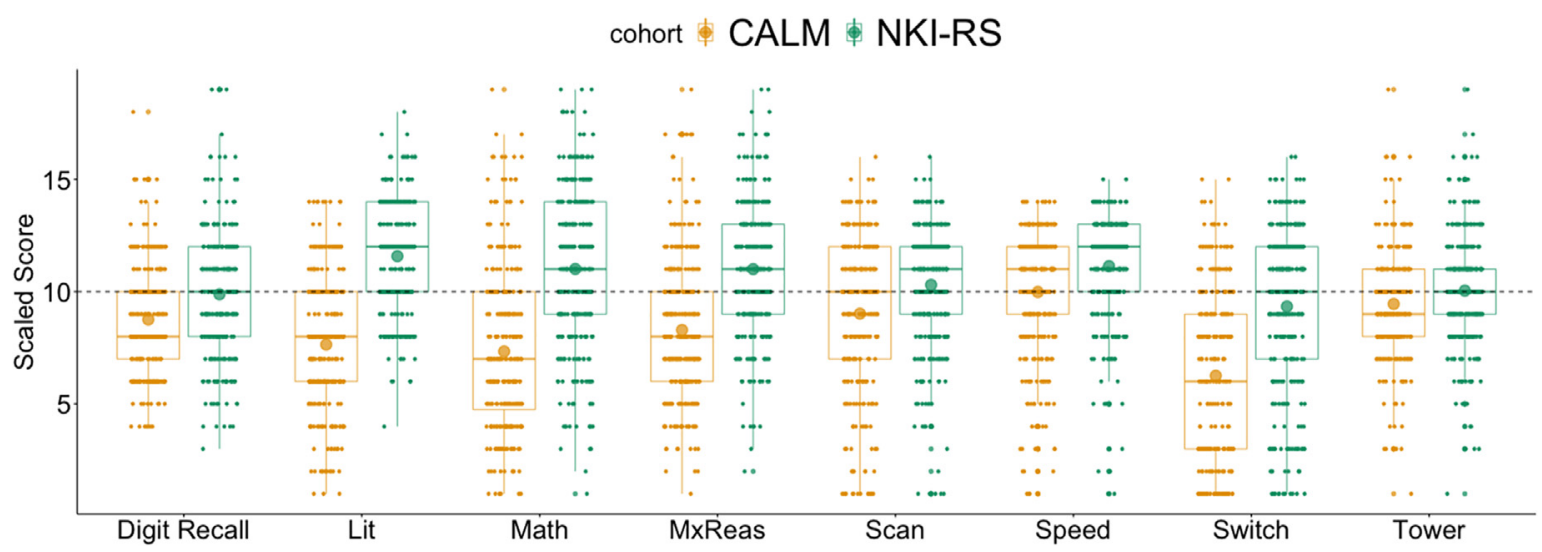

Figure 1. Standardised performance across the tasks for CALM (yellow, left) and NKI-RS (green, right). The large dots inside the boxplots show the mean in each cohort. The grey dotted line represents the age-expected mean. Norm-referenced scores were a mix of T-scores, standard scores, and scaled scores. All scores were converted to scaled scores for visualisation. Digit Recall = WISC-R/AWMA Combined forward and backward digit recall; Lit = Literacy $($ WIAT-II Word reading and spelling); Maths = WIAT-II Numerical operations; MxReas = WASI-II Matrix reasoning; Scan = D-KEFS Visual scanning; Speed = D-KEFS Motor speed; Switch = D-KEFS Trails number-letter sequencing task; Tower = D-KEFS Tower achievement score. (For interpretation of the references to colour in this figure legend, the reader is referred to the web version of this article.)

\section{CALM}

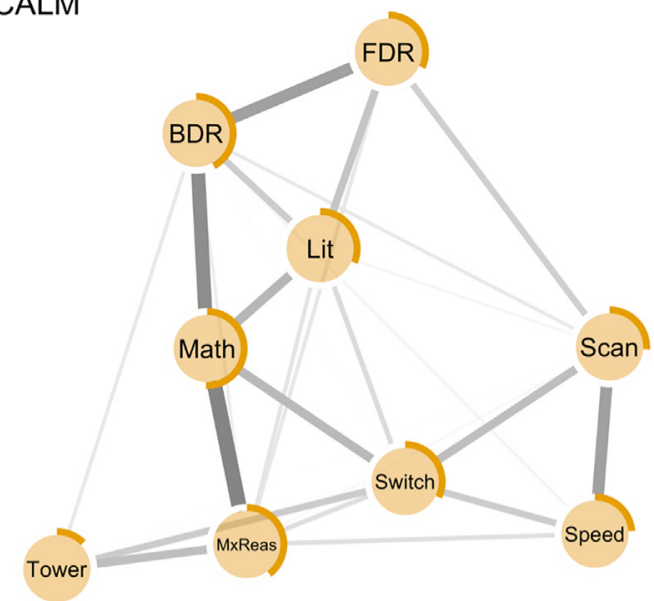

NKI-RS

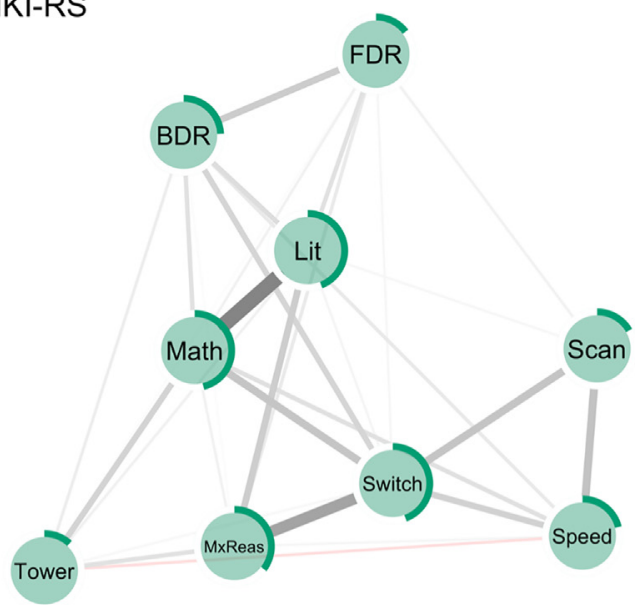

Figure 2. Regularised partial correlation networks across cognitive and academic skills for CALM (left) and NKI-RS (right). Age was included in the estimation but is omitted from figures. Thicker edges represent stronger associations. Red edges reflect negative associations. The rings around the nodes represent proportion of variance explained in the respective node by all connected nodes. Digit Recall $=$ WISC-R/AWMA Combined forward and backward digit recall; Lit $=$ Literacy (WIAT-II Word reading and spelling); Maths = WIAT-II Numerical operations; MxReas = WASI-II Matrix reasoning; Scan = D-KEFS Visual scanning; Speed = DKEFS Motor speed; Switch = D-KEFS Trails number-letter sequencing task; Tower = D-KEFS Tower achievement score. (For interpretation of the references to colour in this figure legend, the reader is referred to the web version of this article.)

imputations via chain equations. Both approaches produced similar outcomes and are fully described in Supplement Section 3.

\section{Results}

\section{Descriptive Statistics}

Raw scores for each task are presented in Table 1 (for normreferenced scores, see Figure 1). Following an adjustment for multiple comparisons, children in NKI-RS significantly outperformed CALM participants across all measures (Table S1). Pearson correlations across raw and age-regressed scores were all positive and are reported in the supplement (Figures S1 and S2). For most tasks, there were significant differences in variance between the groups (see supplement Table S3). As a robustness check, all analyses were repeated following a data transformation, which significantly reduced the differences in variance. The analyses of transformed data produced similar outcomes and are reported in the supplement (see Section 4, Table S3).

\section{Network Estimation and Stability}

A regularised partial correlation network was estimated for each cohort. In both cohorts, the analysis was underpowered to detect age moderation effects on task interrelationships of the magnitude typically reported in the literature (see supplement Section 5). Age was therefore included in the estimation but was omitted from plots and the calculation of centrality indices. In the final networks, nodes represented task performance and edge weights corresponded to the regularised partial 
correlation coefficient between any two tasks, controlling for age and all other scores. Each network was estimated using the graphical variant of the least absolute shrinkage and selection operator with Extended Bayesian Information Criterion used for model selection (Epskamp et al., 2018). The estimated networks are displayed in Figure 2. The bootstrapped 95\% confidence intervals were small to moderate, suggesting acceptable stability (see Figures S5-11, supplement Section 6).

\section{Network Comparison}

As a first step to comparing the networks, the correlation between edge weights across networks was estimated. The correlation was $r_{\mathrm{s}}=.58$, indicating moderate similarity. Second, a permutation test based on 1000 iterations was used to investigate differences in network organisation (van Borkulo et al., 2017). Global connectivity was operationalised as the sum of all absolute edge weights, and global structure reflected the highest absolute difference between two corresponding edges $(M)$. Permutation testing detected no significant difference in global connectivity, suggesting a similar degree of task interrelatedness in both cohorts (CALM: 4.04; NKI-RS: 4.14; $p=.65)$. However, the network structure was not invariant across cohorts $(M=0.30, p<.001)$. Following a false discovery rate correction, four relations between tasks differed between the cohorts: Matrix reasoning - Maths: $r_{\mathrm{CALM}}=.34$ [.25-.42], $r_{\mathrm{NKI}-\mathrm{RS}}=.04$ [.01-.14], $p<.001$; Literacy - Maths: $r_{\mathrm{CALM}}=.20[.1-.29], r_{\mathrm{NKI}-\mathrm{RS}}=.41[.34-.48], p<.001$; Matrix reasoning - Switching: $r_{\mathrm{CALM}}=.09[.01-.21], r_{\mathrm{NKI}-\mathrm{RS}}=.31$ [.19-.40], $p=.022$; Maths - Backward digit span: $r_{\mathrm{CALM}}=.31$ $[.21-.39], r_{\mathrm{NKI}-\mathrm{RS}}=.10[.01-.21], p=.027$. The latter difference was not significant following the data transformation applied to address differences in task variance: $r_{\mathrm{CALM}}=.20, r_{\text {- }}$ $N K I-R S=.06, p=.27$. Alternative network comparisons methods provided similar results and are reported in the supplement (see Sections 7-8, Figures S12-13, and Table S4). Finally, to compare how tasks clustered together across cohorts, a community detection algorithm was applied to each network. In both cohorts, it provided weak evidence for robust task clustering (see supplement Section 9).

\section{Node Centrality and Predictability}

To explore and compare the relative importance of nodes within each network, node strength and predictability were estimated. Strength estimates, defined as the sum of all edge weights connected to a given node, are presented in Figure S14 (see supplement Section 10 for robustness checks). Across cohorts, node strength was strongly correlated $\left(r_{\mathrm{s}}=.75\right.$, $p=.03$ ) and permutation tests suggested no significant differences. Node predictability is the proportion of shared variance between a given task and all tasks related to it (Haslbeck \& Waldorp, 2020). It was estimated by averaging the results of graphical models fitted to each of the 100 imputed datasets per cohort. Nodewise predictability is displayed in Figure 2. Across cohorts, average predictability estimates were similar (CALM: 32\%; NKI-RS: 29\%) and strongly correlated $\left(r_{\mathrm{s}}=.70, p=.04\right)$.

\section{Discussion}

The current study provides one of the first demonstrations of how network approaches can be used to model the complex relationships between cognitive and academic skills proposed by contemporary developmental theories. Associations between these two domains were compared across two developmental cohorts with different levels of ability. One cohort consisted of children referred by health and educational professionals for difficulties related to learning. Their performance was significantly poorer across all tasks relative to the other cohort, which was nationally representative. Despite substantial differences in performance across cohorts, the patterns of task interrelations were broadly similar. There were multiple direct links across cognitive and academic abilities for both cohorts: The matrix reasoning, backward digit recall, and switching tasks were directly related to both literacy and maths. The presence of multiple direct links is consistent with mutualistic and multiple deficit theories (McGrath et al., 2020; Peng \& Kievit, 2020) and provides evidence against specific one-to-one mappings between a single cognitive ability and a specific academic skill.

Several differences in task interrelationships were observed across cohorts. The association between maths and literacy was stronger in the community sample than in the struggling learners. Within a mutualistic perspective, education simultaneously enhances maths and literacy knowledge, and over time these become mutually enriching (Ritchie \& Tucker-Drob, 2018). In struggling learners, weaker skills in one domain might slow the accumulation of knowledge and fluency, and over time, limit the mutually beneficial exchanges between domains. Additionally, negative feedback and/or experiences in one domain may lead to school disengagement or poor motivation for learning, which could have consequences beyond the affected domain (Elliot \& McGregor, 2001; Wery \& Thomson, 2013).

The association between maths and matrix reasoning was stronger in the struggling learners. Through a mutualistic lens, the link between maths and fluid abilities reflects both the benefits of executive abilities for learning maths and the training effects of maths practice on these cognitive abilities (Peng et al., 2019). Learning maths is executively demanding, but practice makes maths knowledge fluent and more easily available for direct retrieval (Mussolin \& Noel, 2008). The stronger link in struggling learners could potentially be due to weaker maths fluency, in which case automatic solution strategies such as direct retrieval will not be available, and mathematical problem solving will instead more strongly draw on executive resources.

Finally, the relationship between the matrix reasoning and switching tasks was weaker in the poor learners. Both tasks are typically considered measures of executive abilities. However, for switching task performance to rely on higher-order cognitive control, children need to be fluent in both the alphabet and counting: Insufficient automatization of the alphabet can falsely impair performance on this task (Egeland \& Follesø, 2020). Therefore, one explanation for the weaker link between tasks is the possibility that in struggling learners, poor 
alphanumeric knowledge constrained task performance more than executive control.

\section{Limitations and Future Directions}

The available data constrains the inferences that can be drawn from the current study. First, we did not capture the full breadth of children's cognitive skills and academic performance because we were limited by the measures available in both cohorts. The inclusion of broader assessments, such as reading comprehension and text-based maths problems, remains an important avenue for future work. Second, the two cohorts were drawn from different countries. Despite broad similarities in the educational systems and cultural values of these countries, it remains possible that the observed differences were due to differences in demographic or school curricula factors across cohorts. Finally, longitudinal data are needed to understand whether the differences observed across cohorts arise because certain abilities operate as a bottleneck to the development of the wider cognitive system, emerge due to different environmental or educational experiences, or a combination of the two.

This study was exploratory but demonstrates that network approaches may offer great value for the field of developmental science. They enable the simultaneous modelling of multiple routes to a specific outcome and can incorporate multidirectional interactions across levels of description. They can therefore be used to test competing theories about how abilities are related across groups and/or time points, and to characterise how genetic, neural, and environmental factors influence these relationships (e.g., Isvoranu et al., 2020; Simpson-Kent et al., 2021). Such applications will be crucial for building an understanding of not only how abilities are related but also which mechanisms enable their wiring.

\section{Conclusion}

Using network science to model and compare the relationships between academic and cognitive skills across cohorts of children with different levels of ability, we find multiple and largely similar interrelationships, together with some key differences. These differences suggest that weak (or absent) reciprocal links between and within academic and cognitive domains may contribute to learning difficulties. Crucially, we demonstrate the potential value of network models for characterising the wiring of cognitive-academic systems across populations. Mutualistic networks provide a promising new tool for capturing complexity in development, and in time may be useful for identifying time windows where interventions could enhance mutualistic coupling.

\section{Conflict of Interest}

The authors declare that they have no conflict of interest.

\section{Acknowledgements}

The Centre for Attention Learning and Memory (CALM) research clinic is based at and supported by funding from the
MRC Cognition and Brain Sciences Unit (CBSU), University of Cambridge. The lead investigators are Joni Holmes, Susan Gathercole, Duncan Astle, Tom Manly, Kate Baker, and Rogier Kievit. Data collection is assisted by a team of researchers and PhD students at the CBSU that includes Joe Bathelt, Giacomo Bignardi, Sarah Bishop, Erica Bottacin, Lara Bridge, Annie Bryant, Sally Butterfield, Elizabeth Byrne, Gemma Crickmore, Fánchea Daly, Tina Emery, Grace Franckel, Laura Forde, Delia Fuhrmann, Andrew Gadie, Sara Gharooni, Jacalyn Guy, Erin Hawkins, Agniezska Jaroslawska, Sara Joeghan, Amy Johnson, Jonathan Jones, Elise Ng-Cordell, Sinéad O’Brien, Cliodhna O'Leary, Joseph Rennie, Ivan SimpsonKent, Roma Siugzdaite, Tess Smith, Stepheni Uh, Francesca Woolgar, and Mengya Zhang. The authors wish to thank the many professionals working in children's services in the South-East and East of England for their support, and to the children and their families for giving up their time to visit the CALM clinic. We would also like to thank all NKIRockland participants and researchers. We would like to thank Dr Jacalyn Guy for reading and commenting on earlier versions of the manuscript. Finally, we thank the anonymous reviewers for their feedback.

\section{Data Availability Statement}

The CALM dataset is not yet available as the study is still ongoing. The data will be made available via managed open access once the study is complete. The NKI-RS dataset is freely available from the Collaborative Informatics and Neuroimaging Suite (COINS). Analysis scripts are available from the corresponding author upon request.

\section{Online Supplement}

Supplementary data to this article can be found online at https://doi.org/10.1016/j.jarmac.2021.08.005.

\section{References}

Alloway, T. P. (2007). Automated working memory assessment: Manual. Pearson.

Altemeier, L. E., Abbott, R. D., \& Berninger, V. W. (2008). Executive functions for reading and writing in typical literacy development and dyslexia. Journal of Clinical and Experimental Neuropsychology, 30(5), 588-606. https://doi.org/10.1080/13803390701562818.

Astle, D. E., Bathelt, J., \& Holmes, J. (2019). Remapping the cognitive and neural profiles of children who struggle at school. Developmental Science, 22(1), 1-17. https://doi.org/10.1111/ desc. 12747.

Astle, D. E., \& Fletcher-Watson, S. (2020). Beyond the core-deficit hypothesis in developmental disorders. Current Directions in Psychological Science, 29(5), 431-437. https://doi.org/10.1177/ 0963721420925518.

Astle, D., Holmes, J., Kievit, R., \& Gathercole, S. E. (2021). The transdiagnostic revolution in neurodevelopmental disorders. Journal of Child Psychology and Psychiatry. Advance online publication. https://doi.org/10.1111/jcpp.13481.

Bishop, D. V. M., \& Snowling, M. J. (2004). Developmental dyslexia and specific language impairment: Same or different? Psycholog- 
ical Bulletin, 130(6), 858-886. https://doi.org/10.1037/00332909.130.6.858.

Booth, J. N., Boyle, J. M. E., \& Kelly, S. W. (2010). Do tasks make a difference? Accounting for heterogeneity of performance of children with reading difficulties on tasks of executive function: Findings from a meta-analysis. British Journal of Developmental Psychology, 28(1), 133-176. https://doi.org/10.1348/ 026151009 X485432.

Borsboom, D. (2016). A network theory of mental disorders. World Psychiatry, 16(1), 5-13. https://doi.org/10.1002/wps.20375.

Bowyer-Crane, C., Snowling, M. J., Duff, F. J., Fieldsend, E., Carroll, J. M., Miles, J., Götz, K., \& Hulme, C. (2008). Improving early language and literacy skills: Differential effects of an oral language versus a phonology with reading intervention. Journal of Child Psychology and Psychiatry and Allied Disciplines, 49(4), 422-432. https://doi.org/10.1111/j.1469-7610.2007.01849.x.

Cain, K., Oakhill, J., \& Lemmon, K. (2004). Individual differences in the inference of word meanings from context: The influence of reading comprehension, vocabulary knowledge, and memory capacity. Journal of Educational Psychology, 96(4), 671-681.

Casey, B. J., Oliveri, M. E., \& Insel, T. (2014). A neurodevelopmental perspective on the research domain criteria (RDoC) framework. Biological Psychiatry, 76(5), 350-353. https://doi.org/10.1016/j. biopsych.2014.01.006.

Castles, A., \& Friedmann, N. (2014). Developmental dyslexia and the phonological deficit hypothesis. Mind and Language, 29(3), 270285. https://doi.org/10.1111/mila.12050.

Clayton, F. J., West, G., Sears, C., Hulme, C., \& Lervåg, A. (2020). A longitudinal study of early reading development: Letter-sound knowledge, phoneme awareness and RAN, but not letter-sound integration, predict variations in reading development. Scientific Studies of Reading, 24(2), 91-107. https://doi.org/10.1080/ 10888438.2019.1622546.

de Beer, J., Engels, J., Heerkens, Y., \& van der Klink, J. (2014). Factors influencing work participation of adults with developmental dyslexia: A systematic review. BMC Public Health, 14(1), 77. https://doi.org/10.1186/1471-2458-14-77.

Delis, D. C., Kaplan, E., \& Kramer, J. H. (2001). Delis-Kaplan executive function system (D-KEFS). The Psychological Corporation.

Egeland, J., \& Follesø, K. (2020). Offering alphabet support in the Trail Making Test: Increasing validity for participants with insufficient automatization of the alphabet. Applied Neuropsychology:Adult, 1-8, Advance online publication. https://doi.org/ 10.1080/23279095.2020.1774377.

Elliot, A. J., \& McGregor, H. A. (2001). A 2 x 2 goal achievement framework. Journal of Personality and Social Psychology, 80(3), 501-519.

Epskamp, S., Borsboom, D., \& Fried, E. I. (2018). Estimating psychological networks and their accuracy: A tutorial paper. Behavior Research Methods, 50(1), 195-212. https://doi.org/ 10.3758/s13428-017-0862-1.

Ferrer, E., McArdle, J. J., Shaywitz, B. A., Holahan, J. M., Marchione, K., \& Shaywitz, S. E. (2007). Longitudinal models of developmental dynamics between reading and cognition from childhood to adolescence. Developmental Psychology, 43(6), 1460-1473. https://doi.org/10.1037/0012-1649.43.6.1460.

Ferrer, E., Shaywitz, B. A., Holahan, J. M., Marchione, K., \& Shaywitz, S. E. (2010). Uncoupling of reading and iq over time: Empirical evidence for a definition of dyslexia. Psychological
Science, 21(1), 0956797609354084.

$93-101$.

https://doi.org/10.1177/

Gathercole, S. E., Pickering, S. J., Knight, C., \& Stegmann, Z. (2004). Working memory skills and educational attainment: Evidence from national curriculum assessments at 7 and 14 years of age. Applied Cognitive Psychology, 18(1), 1-16. https://doi.org/10.1002/ acp.934.

Geary, D. C. (2011). Cognitive predictors of achievement growth in mathematics: A 5-year longitudinal study. Developmental Psychology, 47(6), 1539-1552. https://doi.org/10.1037/a0025510.

Green, C. T., Bunge, S. A., Briones Chiongbian, V., Barrow, M., \& Ferrer, E. (2017). Fluid reasoning predicts future mathematical performance among children and adolescents. Journal of Experimental Child Psychology, 157, 125-143. https://doi.org/10.1016/ j.jecp.2016.12.005.

Haslbeck, J. M. B., \& Waldorp, L. J. (2020). mgm: Estimating TimeVarying Mixed Graphical Models in High-Dimensional Data. Journal of Statistical Software, 93(8), 1-46. https://doi.org/ 10.18637/jss.v093.i08.

Ho, D. E., Imai, K., King, G., \& Stuart, E. A. (2011). MatchIt: Nonparametric preprocessing for parametric causal inference. Journal of Statistical Software, 42, 1-28. http://www.jstatsoft.org/

Holmes, J., Bryant, A., \& Gathercole, S. E. (2019). Protocol for a transdiagnostic study of children with problems of attention, learning and memory (CALM). BMC Pediatrics, 19(1), 10. https:// doi.org/10.1186/s12887-018-1385-3.

Holmes, J., Guy, J., Kievit, R. A., Bryant, A., Mareva, S., CALM Team, \& Gathercole, S. E. (2020). Cognitive dimensions of learning in children with problems in attention, learning and memory. Journal of Educational Psychology. Advance online publication. https://doi.org/10.1037/edu0000644.

Huettig, F., Lachmann, T., Reis, A., \& Petersson, K. M. (2018). Distinguishing cause from effect-many deficits associated with developmental dyslexia may be a consequence of reduced and suboptimal reading experience. Language, Cognition and Neuroscience, 33(3), 333-350. https://doi.org/10.1080/ 23273798.2017 .1348528$.

Isvoranu, A. M., Guloksuz, S., Epskamp, S., Van Os, J., \& Borsboom, D. (2020). Toward incorporating genetic risk scores into symptom networks of psychosis. Psychological Medicine, 50(4), 636-643. https://doi.org/10.1017/S003329171900045X.

Karmiloff-Smith, A. (2009). Nativism versus neuroconstructivism: Rethinking the study of developmental disorders. Developmental Psychology, 45(1), 56-63. https://doi.org/10.1037/a0014506.

Kievit, R. A. (2020). Sensitive periods in cognitive development: A mutualistic perspective. Current Opinion in Behavioral Sciences, 36, 144-149. https://doi.org/10.1016/j.cobeha.2020.10.007.

Kievit, R. A., Lindenberger, U., Goodyer, I. M., Jones, P. B., Fonagy, P., \& Bullmore, E. T., et al. (2017). Mutualistic coupling between vocabulary and reasoning supports cognitive development during late adolescence and early adulthood. Psychological Science, 28(10), 1419-1431. https://doi.org/10.1177/ 0956797617710785.

Kievit, R. A., Hofman, A. D., \& Nation, K. (2019). Mutualistic coupling between vocabulary and reasoning in young children: A replication and extension of the study by Kievit et al. (2017). Psychological Science, 30(8), 1245-125. https://doi.org/10.1177/ 0956797619841265.

Mareva, S., \& Holmes, J. (2019). Transdiagnostic associations across communication, cognitive, and behavioural problems in a 
developmentally at-risk population: A network approach. $B M C$ Pediatrics, 19(1). https://doi.org/10.1186/s12887-019-1818-7.

Mayes, S. D., \& Calhoun, S. L. (2007). Learning, attention, writing, and processing speed in typical children and children with ADHD, autism, anxiety, depression, and oppositional-defiant disorder. Child Neuropsychology, 13(6), 469-493. https://doi.org/10.1080/ 09297040601112773.

McGrath, L. M., Pennington, B. F., Shanahan, M. A., SanterreLemmon, L. E., Barnard, H. D., Willcutt, E. G., Defries, J. C., \& Olson, R. K. (2011). A multiple deficit model of reading disability and attention-deficit/ hyperactivity disorder: Searching for shared cognitive deficits. Journal of Child Psychology and Psychiatry and Allied Disciplines, 52(5), 547-557. https://doi.org/10.1111/j.14697610.2010.02346.x.

McGrath, L. M., Peterson, R. L., \& Pennington, B. F. (2020). The multiple deficit model: Progress, problems, and prospects. Scientific Studies of Reading, 24(1), 7-13. https://doi.org/10.1080/ 10888438.2019.1706180.

Melby-Lervåg, M., Lyster, S. A. H., \& Hulme, C. (2012). Phonological skills and their role in learning to read: A meta-analytic review. Psychological Bulletin, 138(2), 322-352. https://oi.org/10.1037/ a0026744.

Miller-Cotto, D., \& Byrnes, J. P. (2020). What's the best way to characterize the relationship between working memory and achievement?: An initial examination of competing theories. Journal of Educational Psychology, 112(5), 1074-1084. https:// doi.org/10.1037/edu0000395.

Mussolin, C., \& Noel, M.-P. (2008). Specific retrieval deficit from long-term memory in children with poor arithmetic facts abilities. The Open Psychology Journal, 1(1), 26-34. https://doi.org/ 10.2174/1874350100801010026.

Nation, K., \& Hulme, C. (2011). Learning to read changes children's phonological skills: Evidence from a latent variable longitudinal study of reading and nonword repetition. Developmental Science, 14 (4), 649-659. https://doi.org/10.1111/j.1467-7687.2010.01008.x.

Nooner, K. B., Colcombe, S. J., Tobe, R. H., Mennes, M., Benedict, M. M., Moreno, A. L., Panek, L. J., Brown, S., Zavitz, S. T., Li, Q., Sikka, S., Gutman, D., Bangaru, S., Schlachter, R. T., Kamiel, S. M., Anwar, A. R., Hinz, C. M., Kaplan, M. S., Rachlin, A. B., \& Milham, M. P. (2012). The NKI-Rockland Sample: A Model for Accelerating the Pace of Discovery Science in Psychiatry. Frontiers in Neuroscience, 6, 152. https://doi.org/10.3389/ fnins.2012.00152.

Peng, P., \& Fuchs, D. (2016). A meta-analysis of working memory deficits in children with learning difficulties: Is there a difference between verbal domain and numerical domain? Journal of Learning Disabilities, 49(1), 3-20. https://doi.org/10.1177/ 0022219414521667.

Peng, P., \& Kievit, R. A. (2020). The development of academic achievement and cognitive abilities: A bidirectional perspective. Child Development Perspectives, 14(1), 15-20. https://doi.org/ 10.1111/cdep.12352.

Peng, P., Wang, T., Wang, C. C., \& Lin, X. (2019). A meta-analysis on the relation between fluid intelligence and reading/ mathematics: Effects of tasks, age, and social economics status. Psychological Bulletin, 145(2), 189-236. https://doi.org/10.1037/ bul0000182.

Pennington, B. F. (2006). From single to multiple deficit models of developmental disorders. Cognition, 101(2), 385-413. https://doi. org/10.1016/j.cognition.2006.04.008.
Pennington, B. F., \& Ozonoff, S. (1996). Executive functions and developmental psychopathology. Journal of Child Psychology and Psychiatry, 37(1), 51-87. https://doi.org/10.1111/j.14697610.1996.tb01380.x.

Perfetti, C. (2007). Reading ability: Lexical quality to comprehension. Scientific Studies of Reading, 11(4), 357-383. https://doi.org/ 10.1080/10888430701530730.

Peters, L., \& Ansari, D. (2019). Are specific learning disorders truly specific, and are they disorders? Trends in Neuroscience and Education, 17, Article 100115. https://doi.org/10.1016/ j.tine.2019.100115.

Peterson, R. L., Boada, R., McGrath, L. M., Willcutt, E. G., Olson, R. K., \& Pennington, B. F. (2017). Cognitive prediction of reading, math, and attention: Shared and unique influences. Journal of Learning Disabilities, 50(4), 408-421. https://doi.org/10.1177/ 0022219415618500.

Quinn, J. M., Wagner, R. K., Petscher, Y., Roberts, G., Menzel, A. J., \& Schatschneider, C. (2019). Differential codevelopment of vocabulary knowledge and reading comprehension for students with and without learning disabilities. Journal of Educational Psychology, 112(3), 608-627. https://doi.org/10.1037/ edu0000382.

R Core Team. (2020). R: A language and environment for statistical computing. https://www.R-project.org/. https://www.r-project.org/.

Reid, G., \& Fawcett, A. (2008). Dyslexia in context: research, policy and practice. John Wiley \& Sons.

Ritchie, S. J., \& Tucker-Drob, E. M. (2018). How much does education improve intelligence? A meta-analysis. Psychological Science, 29(8), 1358-1369. https://doi.org/10.1177/ 0956797618774253.

Schmitt, S. A., Geldhof, G. J., Purpura, D. J., Duncan, R., \& McClelland, M. M. (2017). Examining the relations between executive function, math, and literacy during the transition to kindergarten: A multi-analytic approach. Journal of Educational Psychology, 109(8), 1120-1140. https://doi.org/10.1037/ edu0000193.

Simpson-Kent, I. L., Fuhrmann, D., Bathelt, J., Achterberg, J., Borgeest, G. S., \& Kievit, R. A. (2020). Neurocognitive reorganization between crystallized intelligence, fluid intelligence and white matter microstructure in two age-heterogeneous developmental cohorts. Developmental Cognitive Neuroscience, 41. https://doi.org/10.1016/J.DCN.2019.100743 100743.

Simpson-Kent, I.L., Fried, E.I., Akarca, D., Mareva, S., Bullmore, E. T., Kievit, R.A., \& CALM Team. (2021). Bridging brain and cognition: A multilayer network analysis of brain structural covariance and general intelligence in a developmental sample of struggling learners. Journal of Intelligence, 9(2), Article 32. https://doi.org/10.3390/jintelligence9020032.

Szucs, D., Devine, A., Soltesz, F., Nobes, A., \& Gabriel, F. (2013). Developmental dyscalculia is related to visuo-spatial memory and inhibition impairment. Cortex, 49(10), 2674-2688. https://doi.org/ 10.1016/j.cortex.2013.06.007.

Taub, G. E., Floyd, R. G., Keith, T. Z., \& McGrew, K. S. (2008). Effects of general and broad cognitive abilities on mathematics achievement. School Psychology Quarterly, 23(2), 187-198. https://doi.org/10.1037/1045-3830.23.2.187.

Tempelaar, W. M., de Vos, N., Plevier, C. M., van Gastel, W. A., Termorshuizen, F., MacCabe, J. H., \& Boks, M. P. M. (2017). Educational level, underachievement, and general mental health 
problems in 10,866 adolescents. Academic Pediatrics, 17(6), 642648. https://doi.org/10.1016/J.ACAP.2017.04.016.

van Borkulo, C. D., Boschloo, L., Kossakowski, J. J., Tio, P., Schoevers, R. A., Borsboom, D., \& Waldorp, L. J. (2017). Comparing network structures on three aspects: A permutation test [Manuscript submitted for publication]. Department of Psychological Methods, University of Amsterdam. https://doi.org/10.13140/ RG.2.2.29455.38569.

Van Der Maas, H. L. J., Dolan, C. V., Grasman, R. P. P. P., Wicherts, J. M., Huizenga, H. M., \& Raijmakers, M. E. J. (2006). A dynamical model of general intelligence: The positive manifold of intelligence by mutualism. Psychological Review, 113(4), 842861. https://doi.org/10.1037/0033-295X.113.4.842.

Van der Ven, S. H. G. G., Kroesbergen, E. H., Boom, J., \& Leseman, P. P. M. M. (2012). The development of executive functions and early mathematics: A dynamic relationship. British Journal of Educational Psychology, 82(1), 100-119. https://doi.org/10.1111/ j.2044-8279.2011.02035.x.

Wechsler, D. (1974).. Wechsler Intelligence Scale for ChildrenRevised (WISC-R). The Psychological Corporation.
Wechsler, D. (2005).. Wechsler Individual Achievement Test II, (2nd ed.). Pearson Assessment.

Wechsler, D. (2011).. Wechsler Abbreviated Scale of Intelligence, (2nd ed.). Pearson Assessment.

Wery, J., \& Thomson, M. M. (2013). Motivational strategies to enhance effective learning in teaching struggling students. Support for Learning, 28(3), 103-108. https://doi.org/10.1111/14679604.12027.

Yeniad, N., Malda, M., Mesman, J., van IJzendoorn, M. H., \& Pieper, S. (2013). Shifting ability predicts math and reading performance in children: A meta-analytical study. Learning and Individual Differences, 23, 1-9. https://doi.org/10.1016/J. LINDIF.2012.10.004.

Received June 1, 2021

Received in revised form August 13, 2021

Accepted August 23, 2021

Available Online: xxxx 